ESAIM: PROCEEDINGS, December 2003, Vol. 13, 18-30

J.P. Penot, Editor

DOI: $10.1051 /$ proc:2003001

\title{
NECESSARY AND SUFFICIENT OPTIMALITY CONDITIONS FOR OPTIMIZATION PROBLEMS IN FUNCTION SPACES AND APPLICATIONS TO CONTROL THEORY*
}

\author{
E. Casas $^{1}$, Mariano Mateos $^{2}$ and Fredi Tröltzsch ${ }^{3}$
}

\begin{abstract}
We consider an abstract formulation for optimization problems in some $L^{p}$ spaces. The variables are restricted by pointwise upper and lower bounds and by finitely many equality and inequality constraints of functional type. Second-order necessary and sufficient optimality conditions are established, where the cone of critical directions is arbitrarily close to the form which is expected from the optimization in finite dimensional spaces. The results are applied to an optimal control problem governed by a partial differential equation. Finally we compare the conditions obtained by applying this abstract procedure and those ones derived by using the methods adapted to the optimal control problem.
\end{abstract}

Résumé. Nous considérons des problèmes d'optimisation formulés de manière abstraite dans des espaces de type $L^{p}$. Les variables doivent vérifier des contraintes ponctuelles de borne supérieure et inférieure, ainsi qu'un nombre fini de contraintes d'égalité et d'inégalité fonctionnelles. Nous établissons des conditions nécessaires et des conditions suffisantes d'optimalité, dans lesquelles le cône des directions critiques est arbitrairement proche de la forme dérivée des résultats d'optimisation en dimension finie.Les résultats sont appliqués à des problèmes de contrôle optimal gouvernés par des équations aux dérivées partielles. Finalement nous comparons ces conditions avec celles obtenues par des méthodes adaptées à la forme spécifique des problèmes de contrôle.

\section{INTRODUCTION}

The goal of this paper is to derive second order optimality conditions for optimal control problems of partial differential equations. While there exists a vast literature about first order optimality conditions, only a few references deal with the second order conditions for optimality. However the sufficient second order conditions are very important to analyze the convergence properties of the numerical optimization algorithms used to solved the control problems; see Alt and Malanowski [1], Dontchev et al. [14] or Ito and Kunisch [19] and the references cited therein. They are also the key tool to obtain the error estimates in the numerical discretization of the control problem; see Arada, Casas and Tröltzsch [2], Casas [6] and Hager [18]. Among the papers devoted to the sufficient optimality conditions let us mention Goldberg and Tröltzsch [17], Casas, Tröltzsch,

\footnotetext{
* The first two authors were partially supported by Ministerio de Ciencia y Tecnología (Spain)

${ }^{1}$ Departamento de Matemática Aplicada y Ciencias de la Computación, E.T.S.I. Industriales y de Telecomunicación, Universidad de Cantabria, 39005 Santander, Spain ,e-mail: eduardo.casas@unican.es

2 Departamento de Matemáticas, EUIT Industriales, Universidad de Oviedo., Spain, e-mail: mmateos@orion.ciencias.uniovi.es

3 Fakultät für Mathematik, Technische Universität Chemnitz, D-09107 Chemnitz, Germany,

e-mail: f.troeltzsch@mathematik.tu-chemnitz.de
} 
and Unger [11], [12], Raymond and Tröltzsch [22]. The question now is why are we interested in the secondorder necessary optimality conditions? The answer is clear. It is not difficult to provide sufficient second order conditions very useful to carry out the numerical analysis of the control problems, but we should ask ourselves if they are realistic in the sense that they are satisfied frequently. The smaller gap between necessary and sufficient optimality conditions the higher probability for the sufficient conditions to be held. Therefore we are concerned with the derivation of necessary and sufficient second order conditions with a minimum gap between them; see Bonnans and Zidani [4], Casas and Tröltzsch [9], Casas, Fernández and Mateos [8]. In this paper we summarize the results proved by the authors in [10] and [7].

We can try two different ways to deduce the second-order conditions. The first procedure consists of applying some abstract methods for optimization to the control problem. This was followed in [10]. A second way can be tried by using some specific results for control problems, using for instance Pontryagin's principle. This second idea was developed in [7]. Both methods lead to apparently different results, but we will prove that they are equivalent.

In $\S 1$ we will formulate an abstract optimization problem well adapted to the control theory. Indeed this formulation is valid for distributed or boundary control problems as well as for problems governed by partial differential equations or ordinary differential equations. In $\S 2$ we follow a technique adapted to the control problem and finally we establish the equivalence between the results obtained by both methods.

\section{An Abstract Optimization Problem}

Let $(X, \mathcal{S}, \mu)$ be a measure space with $\mu(X)<+\infty$. In this paper we will study the following optimization problem

$$
(\mathrm{P})\left\{\begin{array}{l}
\text { Minimize } J(u) \\
u_{a}(x) \leq u(x) \leq u_{b}(x) \text { a.e. } x \in X, \\
G_{j}(u)=0,1 \leq j \leq m_{1}, \\
G_{j}(u) \leq 0, m_{1}+1 \leq j \leq m,
\end{array}\right.
$$

where $u_{a}, u_{b} \in L^{\infty}(X)$ and $J, G_{j}: L^{\infty}(X) \longrightarrow \mathbb{R}$ are given functions with differentiability properties to be fixed later. We will state necessary and sufficient optimality conditions for a local minimum of $(\mathrm{P})$.

Before establishing the results for this problem let us give some examples.

Example 1. A Distributed Control Problem.

where $y_{u}$ is the solution of

$$
(\mathrm{P})\left\{\begin{array}{l}
\text { Minimize } J(u)=\int_{\Omega} L\left(x, y_{u}(x), u(x)\right) d x \\
u_{a}(x) \leq u(x) \leq u_{b}(x) \text { a.e. } x \in \Omega \\
F_{j}\left(y_{u}\right)=0,1 \leq j \leq n_{e} \\
F_{j}\left(y_{u}\right) \leq 0, n_{e}+1 \leq j \leq n_{e}+n_{i}
\end{array}\right.
$$

$$
\left\{\begin{array}{l}
-\Delta y_{u}=f\left(x, y_{u}, u\right) \text { in } \Omega \\
\partial_{\nu} y_{u}=g \text { on } \Gamma
\end{array}\right.
$$

$\Omega \subset \mathbb{R}^{N}, \Gamma$ is the boundary of $\Omega$ of class $C^{1}, g \in L^{p(1-1 / N)}(\Gamma), p>N$, and $u_{a}, u_{b} \in L^{\infty}(\Omega), u_{a}(x) \leq u_{b}(x)$ for a.e. $x \in \Omega$.

It is easy to include this problem in the abstract framework by setting $X=\Omega, \mu=$ Lebesgue measure in $\Omega$ and $G_{j}(u)=F_{j}\left(y_{u}\right)$.

Example 2. A Boundary Control Problem

$$
(\mathrm{P})\left\{\begin{array}{l}
\text { Minimize } J(u) \\
u_{a}(x) \leq u(x) \leq u_{b}(x) \text { a.e. } x \in \Gamma \\
F_{j}\left(y_{u}\right)=0, \quad 1 \leq j \leq m_{1} \\
F_{j}\left(y_{u}\right) \leq 0, \quad m_{1}+1 \leq j \leq m
\end{array}\right.
$$


with

the state equation given by

$$
J(u)=\int_{\Omega} f\left(x, y_{u}(x)\right) d x+\int_{\Gamma} g\left(x, y_{u}(x), u(x)\right) d S(x),
$$

$$
\left\{\begin{array}{l}
-\Delta y(x)+y(x)=0 \text { in } \Omega \\
\partial_{\nu} y(x)=b(x, y(x), u(x)) \text { on } \Gamma,
\end{array}\right.
$$

$F_{j}: C(\bar{\Omega}) \longrightarrow \mathbb{R}$ of class $C^{2}, 1 \leq j \leq m$ and $u_{a}, u_{b} \in L^{\infty}(\Gamma)$, with $u_{a}(x) \leq u_{b}(x)$.

In this example $X=\Gamma$ and $\mu=$ the surface measure on $\Gamma$.

Some other control problems can be included in the above abstract formulation such as control of parabolic equations $(X=\Omega \times(0, T)$ or $X=\Gamma \times(0, T))$ or ordinary differential equations $(X=(0, T))$; see [10] for more examples.

The reader could think that a more abstract formulation could be more interesting. Why have we taken the space $L^{\infty}(X)$ instead a more general Banach space $U$ ? First of all, it is easier to consider the control constraints $u_{a}(x) \leq u(x) \leq u_{b}(x)$ in some $L^{p}$ space and the formulation of some theorems will be clearer in these spaces. $L^{p}$ spaces are well adapted to the formulation of control problems. But there is a second reason not less important. To derive the second order optimality conditions we have to deal with the so-called two-norm discrepancy. The classical necessary and sufficient second order optimality conditions for problems consisting of the minimization of a function $J$ in some Banach space $U$ (see for instance H. Cartan [5]) are not very useful. Let us consider the following example.

Example 3. The two-norm discrepancy.

Let $X=[0,1]$ and $J: L^{2}[0,1] \longrightarrow \mathbb{R}$ defined by

We consider the problem

$$
J(u)=\int_{0}^{1} \sin (u(x)) d x
$$

$$
\text { (P) }\left\{\begin{array}{l}
\text { Minimize } J(u) \\
u \in L^{2}[0,1]
\end{array}\right.
$$

Then $\bar{u}(x)=-\pi / 2$ is obviously a solution. We have

$$
J^{\prime}(\bar{u}) h=\int_{0}^{1} \cos (\bar{u}(x)) h(x) d x=0 \quad \forall h \in L^{2}[0,1]
$$

and

$$
J^{\prime \prime}(\bar{u}) h^{2}=-\int_{0}^{1} \sin (\bar{u}(x)) h^{2}(x) d x=\|h\|_{L^{2}[0,1]}^{2} .
$$

Apparently we can apply the classical sufficient optimality conditions to deduce that $\bar{u}$ is a strict local minimum of $(\mathrm{P})$ in $L^{2}[0,1]$. But it is not. Indeed any function of the form

$$
\bar{u}_{\varepsilon}(x)=\left\{\begin{array}{cc}
3 \pi / 2 & \text { if } x \in[0, \varepsilon] \\
-\pi / 2 & \text { if } x \in(\varepsilon, 1]
\end{array}\right.
$$

is a solution for any $0<\varepsilon<1$. Which is wrong in the above argument? The error comes from the fact that $J$ is not $C^{2}$ in $L^{2}[0,1]$. This function is $C^{2}$ in $L^{\infty}[0,1]$, but not in $L^{2}[0,1]$. However we can prove that $J^{\prime \prime}(\bar{u}) h^{2} \geq\|h\|_{L^{2}[0,1]}^{2}$ but not $J^{\prime \prime}(\bar{u}) h^{2} \geq\|h\|_{L^{\infty}[0,1]}^{2}$. It is clear that the natural norm to be used on the sufficient optimality conditions is the $L^{2}$-norm, but the differentiability requires the $L^{\infty}$-norm. This is the two-norm discrepancy and this the usual case in the applications, we have to deal with these two norms. By using the first norm the functions are twice differentiable ant with the second norm we deduce the sufficient optimality condition to be a strict local minimum in the space endowed with the fist norm. This is the reason for the above abstract framework. 
In the sequel we will assume that $\bar{u}$ is a local solution of the abstract problem $(\mathrm{P})$, which means that there exists a real number $r>0$ such that for every feasible point of $(\mathrm{P})$, with $\|u-\bar{u}\|_{L^{\infty}(X)}<r$, we have that $J(\bar{u}) \leq J(u)$.

For every $\varepsilon>0$, we denote the set of points where the bound constraints are $\varepsilon$-inactive by

$$
X_{\varepsilon}=\left\{x \in X: u_{a}(x)+\varepsilon \leq \bar{u}(x) \leq u_{b}(x)-\varepsilon\right\} .
$$

We make the following regularity assumption

$$
\left\{\begin{array}{l}
\exists \varepsilon_{\bar{u}}>0 \text { and }\left\{h_{j}\right\}_{j \in I_{0}} \subset L^{\infty}(X), \text { with supp } h_{j} \subset X_{\varepsilon_{\bar{u}}}, \text { such that } \\
G_{i}^{\prime}(\bar{u}) h_{j}=\delta_{i j}, \quad i, j \in I_{0},
\end{array}\right.
$$

where

$$
I_{0}=\left\{j \leq m \mid G_{j}(\bar{u})=0\right\}
$$

$I_{0}$ is the set of indices corresponding to active constraints. We also denote the set of non active constraints by $I_{-}$

$$
I_{-}=\left\{j \leq m \mid G_{j}(\bar{u})<0\right\}
$$

Obviously (3) is equivalent to the linear independence of the derivatives $\left\{G_{j}^{\prime}(\bar{u})\right\}_{j \in I_{0}}$ in $L^{\infty}\left(X_{\varepsilon_{\bar{u}}}\right)$. Under this assumption we can derive the first-order necessary conditions for optimality satisfied by $\bar{u}$. For the proof the reader is referred to Bonnans and Casas [3] or Clarke [13].

Theorem 1. Let us assume that (3) holds and $J$ and $\left\{G_{j}\right\}_{j=1}^{m}$ are of class $C^{1}$ in a neighbourhood of $\bar{u}$. Then there exist real numbers $\left\{\bar{\lambda}_{j}\right\}_{j=1}^{m} \subset \mathbb{R}$ such that

$$
\begin{gathered}
\bar{\lambda}_{j} \geq 0, \quad m_{1}+1 \leq j \leq m, \bar{\lambda}_{j}=0 \text { if } j \in I_{-} \\
\left\langle J^{\prime}(\bar{u})+\sum_{j=1}^{m} \bar{\lambda}_{j} G_{j}^{\prime}(\bar{u}), u-\bar{u}\right\rangle \geq 0 \quad \text { for all } u_{a} \leq u \leq u_{b} .
\end{gathered}
$$

In order to deal with the two-norm discrepancy we have to impose some additional assumptions on the functions $J$ and $G_{j}$. The reader can check that they are fulfilled by the control problems of Examples 1 and 2 .

(A1): There exist functions $f, g_{j} \in L^{2}(X), 1 \leq j \leq m$, such that for every $h \in L^{\infty}(X)$

$$
J^{\prime}(\bar{u}) h=\int_{X} f(x) h(x) d \mu(x) \text { and } G_{j}^{\prime}(\bar{u}) h=\int_{X} g_{j}(x) h(x) d \mu(x), 1 \leq j \leq m .
$$

(A2): If $\left\{h_{k}\right\}_{k=1}^{\infty} \subset L^{\infty}(X)$ is bounded, $h \in L^{\infty}(X)$ and $h_{k}(x) \rightarrow h(x)$ a.e. in $X$, then

If we define

$$
\left[J^{\prime \prime}(\bar{u})+\sum_{j=1}^{m} \bar{\lambda}_{j} G_{j}^{\prime \prime}(\bar{u})\right] h_{k}^{2} \rightarrow\left[J^{\prime \prime}(\bar{u})+\sum_{j=1}^{m} \bar{\lambda}_{j} G_{j}^{\prime \prime}(\bar{u})\right] h^{2} .
$$

then

$$
\mathcal{L}(u, \lambda)=J(u)+\sum_{j=1}^{m} \lambda_{j} G_{j}(u) \text { and } d(x)=f(x)+\sum_{j=1}^{m} \bar{\lambda}_{j} g_{j}(x),
$$

$$
\frac{\partial \mathcal{L}}{\partial u}(\bar{u}, \bar{\lambda}) h=\left[J^{\prime}(\bar{u})+\sum_{j=1}^{m} \bar{\lambda}_{j} G_{j}^{\prime}(\bar{u})\right] h=\int_{X} d(x) h(x) d \mu(x) \quad \forall h \in L^{\infty}(X) .
$$

From (5) we deduce that

$$
d(x)=\left\{\begin{array}{cl}
0 & \text { for a.e. } x \in X \text { where } u_{a}(x)<\bar{u}(x)<u_{b}(x) \\
\geq 0 & \text { for a.e. } x \in X \text { where } \bar{u}(x)=u_{a}(x) \\
\leq 0 & \text { for a.e. } x \in X \text { where } \bar{u}(x)=u_{b}(x)
\end{array}\right.
$$


Remark 1. ¿From (5), (9), and assumption (3) we get

$$
\frac{\partial \mathcal{L}}{\partial u}(\bar{u}, \bar{\lambda}) \bar{h}_{i}=\left\langle J^{\prime}(\bar{u})+\sum_{j=1}^{m} \bar{\lambda}_{j} G_{j}^{\prime}(\bar{u}), h_{i}\right\rangle=J^{\prime}(\bar{u}) h_{i}+\bar{\lambda}_{i}=0,
$$

which implies the uniqueness of the Lagrange multipliers provided in Theorem 1.

Associated with $d$ we set

$$
X^{0}=\{x \in X:|d(x)|>0\} .
$$

Given $\left\{\bar{\lambda}_{j}\right\}_{j=1}^{m}$ by Theorem 1 we define the cone of critical directions

$$
C_{\bar{u}}^{0}=\left\{h \in L^{\infty}(X) \text { satisfying (13) and } h(x)=0 \text { for a.e. } x \in X^{0}\right\},
$$

with

$$
\left\{\begin{array}{l}
G_{j}^{\prime}(\bar{u}) h=0 \text { if }\left(j \leq m_{1}\right) \text { or }\left(j>m_{1}, G_{j}(\bar{u})=0 \text { and } \bar{\lambda}_{j}>0\right) ; \\
G_{j}^{\prime}(\bar{u}) h \leq 0 \text { if } j>m_{1}, G_{j}(\bar{u})=0 \text { and } \bar{\lambda}_{j}=0 ; \\
h(x)=\left\{\begin{array}{l}
\geq 0 \text { if } \bar{u}(x)=u_{a}(x) ; \\
\leq 0 \text { if } \bar{u}(x)=u_{b}(x) .
\end{array}\right.
\end{array}\right.
$$

In the following theorem we state the necessary second-order optimality conditions.

Theorem 2. Assume that (3), (A1) and (A2) hold, $\left\{\bar{\lambda}_{j}\right\}_{j=1}^{m}$ are the Lagrange multipliers satisfying (4) and (5) and $J$ and $\left\{G_{j}\right\}_{j=1}^{m}$ are of class $C^{2}$ in a neighbourhood of $\bar{u}$. Then the following inequality is satisfied

$$
\frac{\partial^{2} \mathcal{L}}{\partial u^{2}}(\bar{u}, \bar{\lambda}) h^{2} \geq 0 \quad \forall h \in C_{\bar{u}}^{0}
$$

The proof of this theorem makes use of the following lemma

Lemma 1. Let us assume that (3) holds and $J$ and $\left\{G_{j}\right\}_{j=1}^{m}$ are of class $C^{2}$ in a neighbourhood of $\bar{u}$. Let $h \in L^{\infty}(X)$ satisfy $G_{j}^{\prime}(\bar{u}) h=0$ for every $j \in I$, where $I$ is an arbitrary subset of $I_{0}$. Then there exist a number $\varepsilon_{h}>0$ and $C^{2}$-functions $\gamma_{j}:\left(-\varepsilon_{h},+\varepsilon_{h}\right) \longrightarrow \mathbb{R}, j \in I$, such that

$$
\left\{\begin{array}{l}
G_{j}\left(u_{t}\right)=0 j \in I, \text { and } G_{j}\left(u_{t}\right)<0 j \notin I_{0}, \quad \forall|t| \leq \varepsilon_{h} \\
\gamma_{j}(0)=\gamma_{j}^{\prime}(0)=0, j \in I
\end{array}\right.
$$

with $u_{t}=\bar{u}+t h+\sum_{j \in I} \gamma_{j}(t) h_{j},\left\{h_{j}\right\}_{j \in I}$ given by (3).

See [9] or [10] for the proof.

Motivated again by the considerations on the two-norm discrepancy we have to make some assumptions involving the $L^{\infty}(X)$ and $L^{2}(X)$ norms,

(A3): There exists a positive number $r>0$ such that $J$ and $\left\{G_{j}\right\}_{j=1}^{m}$ are of class $C^{2}$ in the $L^{\infty}(X)$-ball $B_{r}(\bar{u})$ and for every $\eta>0$ there exists $\varepsilon \in(0, r)$ such that for each $u \in B_{r}(\bar{u}),\|v-\bar{u}\|_{L^{\infty}(X)}<\varepsilon$, $h, h_{1}, h_{2} \in L^{\infty}(X)$ and $1 \leq j \leq m$ we have 


$$
\left\{\begin{array}{l}
\left|\left[\frac{\partial^{2} \mathcal{L}}{\partial u^{2}}(v, \bar{\lambda})-\frac{\partial^{2} \mathcal{L}}{\partial u^{2}}(\bar{u}, \bar{\lambda})\right] h^{2}\right| \leq \eta\|h\|_{L^{2}(X)}^{2} \\
\left|J^{\prime}(u) h\right| \leq M_{0,1}\|h\|_{L^{2}(X)}, \quad\left|G_{j}^{\prime}(u) h\right| \leq M_{j, 1}\|h\|_{L^{2}(X)} \\
\left|J^{\prime \prime}(u) h_{1} h_{2}\right| \leq M_{0,2}\left\|h_{1}\right\|_{L^{2}(X)}\left\|h_{2}\right\|_{L^{2}(X)} \\
\left|G_{j}^{\prime \prime}(u) h_{1} h_{2}\right| \leq M_{j, 2}\left\|h_{1}\right\|_{L^{2}(X)}\left\|h_{2}\right\|_{L^{2}(X)}
\end{array}\right.
$$

Analogously to (11) and (12) we define for every $\tau>0$

$$
\begin{gathered}
X^{\tau}=\{x \in X:|d(x)|>\tau\} \\
C_{\bar{u}}^{\tau}=\left\{h \in L^{\infty}(X) \text { satisfying (13) and } h(x)=0 \text { a.e. } x \in X^{\tau}\right\} .
\end{gathered}
$$

The next theorem provides the second-order sufficient optimality conditions of $(\mathrm{P})$. Though they seem to be different from the classical ones, we will see later that they are equivalent; see Theorem 4 and Corollary 1.

Theorem 3 (Casas and Tröltzsch [10]). Let $\bar{u}$ be a feasible point for problem (P) verifying the first-order necessary conditions (4) and (5), and let us suppose that assumptions (3), (A1) and (A3) hold. Let us also assume that for every $h \in L^{\infty}(X)$ satisfying (13)

$$
\frac{\partial^{2} \mathcal{L}}{\partial u^{2}}(\bar{u}, \bar{\lambda}) h^{2} \geq \delta_{1}\|h\|_{L^{2}\left(X \backslash X^{\tau}\right)}^{2}-\delta_{2}\|h\|_{L^{2}\left(X^{\tau}\right)}^{2}
$$

holds for some $\delta_{1}>0, \delta_{2} \geq 0$ and $\tau>0$. Then there exist $\varepsilon>0$ and $\delta>0$ such that $J(\bar{u})+\delta\|u-\bar{u}\|_{L^{2}(X)}^{2} \leq J(u)$ for every feasible point $u$ for $(\mathrm{P})$, with $\|u-\bar{u}\|_{L^{\infty}(X)}<\varepsilon$.

This theorem can be formulated in a more classical way.

Theorem 4 (Casas and Tröltzsch [10]). Let $\bar{u}$ be a feasible point of $(\mathrm{P})$ satisfying $(4)$ and $(5)$. Let $C_{\bar{u}}$ be the set of elements $h \in L^{\infty}(X)$ satisfying (13) and $C_{\bar{u}}^{\tau}$ be given by (18). Let us suppose that assumptions (3), (A1) and (A3) hold. Let $\tau>0$ be given. Then the following statements are equivalent

$$
\begin{aligned}
\exists \delta>0 \quad: \quad \frac{\partial^{2} \mathcal{L}}{\partial u^{2}}(\bar{u}, \bar{\lambda}) h^{2} \geq \delta\|h\|_{L^{2}(X)}^{2} \quad \forall h \in C_{\bar{u}}^{\tau}, \\
\exists \delta_{1}>0, \delta_{2} \geq 0 \quad: \quad \frac{\partial^{2} \mathcal{L}}{\partial u^{2}}(\bar{u}, \bar{\lambda}) h^{2} \geq \delta_{1}\|h\|_{L^{2}\left(X \backslash X^{\tau}\right)}^{2}-\delta_{2}\|h\|_{L^{2}\left(X^{\tau}\right)}^{2} \quad \forall h \in C_{\bar{u}} .
\end{aligned}
$$

The following corollary is an immediate consequence of Theorems 3 and 4.

Corollary 1. Let $\bar{u}$ be a feasible point for problem (P) satisfying (4) and (5) and let us suppose that assumptions (3), (A1) and (A3) hold. Let us also assume that

$$
\frac{\partial^{2} \mathcal{L}}{\partial u^{2}}(\bar{u}, \bar{\lambda}) h^{2} \geq \delta\|h\|_{L^{2}(X)}^{2} \quad \forall h \in C_{\bar{u}}^{\tau}
$$

for some $\delta>0$ and $\tau>0$ given. Then there exist $\varepsilon>0$ and $\alpha>0$ such that $J(\bar{u})+\alpha\|u-\bar{u}\|_{L^{2}(X)}^{2} \leq J(u)$ for every feasible point $u$ for $(\mathrm{P})$, with $\|u-\bar{u}\|_{L^{\infty}(X)}<\varepsilon$.

Remark 2. Comparing the sufficient optimality condition (22) with the necessary one (14), we notice the existence of a gap between both coming from two facts. Firstly the constant $\delta$ is strictly positive in (19) and it can be zero in (14), which is the classical situation even in finite dimension. The second fact is that we can not replace, in general, $C_{\bar{u}}^{\tau}$, with $\tau>0$, for $C_{\bar{u}}^{0}$ in (22), as it is done in (14). This is motivated by the 
presence of an infinite number of constraints. Quite similar strategies are employed by Maurer and Zowe [21], Maurer [20], Donchev, Hager, Poore and Yang [14] and Dunn [15]. The following example, due to J.C. Dunn [16], demonstrates the impossibility of taking $\tau=0$ in (22). Let us consider $X=[0,1], \mathcal{S}$ the $\sigma$-algebra of Lebesgue measurable sets of $[0,1], \mu$ the Lebesgue measure in $[0,1]$ and $a(x)=1-2 x$. The optimization problem is

$$
\left\{\begin{array}{l}
\text { Minimize } J(u)=\int_{0}^{1}\left[2 a(x) u(x)-\operatorname{sign}(a(x)) u(x)^{2}\right] d x \\
u \in L^{\infty}[0,1], u(x) \geq 0 \text { a.e. } x \in[0,1]
\end{array}\right.
$$

Let us set $\bar{u}(x)=\max \{0,-a(x)\}$. Then we have

$$
J^{\prime}(\bar{u}) h=\int_{0}^{1} 2[a(x)-\operatorname{sign}(a(x)) \bar{u}(x)] h(x) d x=\int_{0}^{1 / 2} 2 a(x) h(x) d x \geq 0
$$

holds for all $h \in L^{2}([0,1])$, with $h(x) \geq 0$. If we assume that $h(x)=0$ for $x \in X^{0}$,

$$
J^{\prime \prime}(\bar{u}) h^{2}=-\int_{0}^{1} 2 \operatorname{sign}(a(x)) h^{2}(x) d x=2 \int_{1 / 2}^{1} h^{2}(x) d x-2 \int_{0}^{1 / 2} h^{2}(x) d x=2\|h\|_{L^{2}(X)}^{2}
$$

holds, where, following the notation introduced in (11),

$$
X^{0}=\{x \in[0,1]:|d(x)|>0\}=[0,1 / 2)
$$

Thus (22) holds with $\delta=2$ and $\tau=0$. However $\bar{u}$ is not a local minimum in $L^{\infty}[0,1]$. Indeed, let us take for $0<\varepsilon<\frac{1}{2}$

$$
u_{\varepsilon}(x)=\left\{\begin{array}{cl}
\bar{u}(x)+3 \varepsilon & \text { if } x \in\left[\frac{1}{2}-\varepsilon, \frac{1}{2}\right] \\
\bar{u}(x) & \text { otherwise. }
\end{array}\right.
$$

Then we have $J\left(u_{\varepsilon}\right)-J(\bar{u})=-3 \varepsilon^{3}<0$. The reader can easily check that the only points $u$ satisfying the first order optimality conditions are given by the formula

$$
u(x)=\left\{\begin{array}{cl}
0 & \text { if } x \in Z \\
\operatorname{sign}(a(x)) a(x) & \text { otherwise }
\end{array}\right.
$$

where $Z$ is any measurable subset of $[0,1]$ satisfying that $a(x) \geq 0$ for every $x \in Z$. None of these points is a local minimum of the optimization problem. Even more, if we define $u_{k}(x)=k \cdot \max \{0, a(x)\}$, then $J\left(u_{k}\right)=k(2-k) / 6 \rightarrow-\infty$ when $k \rightarrow+\infty$.

It seems quite natural to ask if the cones $C_{\bar{u}}^{\tau}, \tau \geq 0$, can be taken in $L^{2}(X)$ instead of $L^{\infty}(X)$. The answer is provided by the following theorem.

Theorem 5 (Casas and Mateos [7]). $C_{\bar{u}, L^{2}(X)}^{\tau}=$ the closure of $C_{\bar{u}}^{\tau}$ in $L^{2}(X)$.

Now it is enough to assume that

$$
\frac{\partial^{2} \mathcal{L}}{\partial u^{2}}(\bar{u}, \bar{\lambda}) h_{k}^{2} \longrightarrow \frac{\partial^{2} \mathcal{L}}{\partial u^{2}}(\bar{u}, \bar{\lambda}) h^{2}
$$

whenever $h_{k} \rightarrow h$ in $L^{2}(X)$ to deduce from Theorem 5 that we can replace $C_{\bar{u}}^{\tau}$ by $C_{\bar{u}, L^{2}(X)}^{\tau}$ in $(22)$. In particular this assumption is fulfilled by the control problems given in Examples 1 and 2 . 


\section{Application to the distributed optimal Control problem}

In this section we apply the results of the previous section to the distributed control problem considered in Example 1. We could do the same for the problem described in Example 2. Let us precise the assumptions on the control problem.

(H1) $f$ is of class $C^{2}$ with respect to the second and third variables,

$$
f(\cdot, 0,0) \in L^{N p /(N+p)}(\Omega), \quad \frac{\partial f}{\partial y}(x, y, u) \leq 0,
$$

and for all $M>0$ there exists a constant $C_{f, M}>0$ such that

$$
\left|\frac{\partial f}{\partial y}(x, y, u)\right|+\left|\frac{\partial f}{\partial u}(x, y, u)\right|+\left|\frac{\partial^{2} f}{\partial y^{2}}(x, y, u)\right|+\left|\frac{\partial^{2} f}{\partial y \partial u}(x, y, u)\right|+\left|\frac{\partial^{2} f}{\partial u^{2}}(x, y, u)\right| \leq C_{f, M}
$$

for a.e. $x \in \Omega$ and $|y|,|u| \leq M$. Remind that $p>N$ is given in the formulation of Example 1. Moreover, given $\rho>0$ arbitrary, for every $\varepsilon>0$ there exists $\delta>0$ such that for almost every point $x \in \Omega$ and $\left|y_{i}\right|,\left|u_{i}\right| \leq \rho$, $i=1,2$, we assume

$$
\left|D_{(y, u)}^{2} f\left(x, y_{2}, u_{2}\right)-D_{(y, u)}^{2} f\left(x, y_{1}, u_{1}\right)\right|<\varepsilon \text { if }\left|y_{2}-y_{1}\right|<\delta,\left|u_{2}-u_{1}\right|<\delta
$$

where $D_{(y, u)}^{2} f$ denotes the second derivative of $f$ with respect to $(y, u)$.

(H2) $L: \Omega \times \mathbb{R} \times \mathbb{R} \longrightarrow \mathbb{R}$ is of class $C^{2}$ with respect to the second and third variables, $|L(\cdot, 0,0)| \in L^{1}(\Omega)$, and for all $M>0$ there exists a constant $C_{M}>0$ and functions $\psi_{M} \in L^{N p /(N+p)}(\Omega)$ and $\psi_{M}^{*} \in L^{2}(\Omega)$ such that

and

$$
\left|\frac{\partial L}{\partial y}(x, y, u)\right| \leq \psi_{M}(x),\left|\frac{\partial L}{\partial u}(x, y, u)\right| \leq \psi_{M}^{*}(x)
$$

$$
\left|\frac{\partial^{2} L}{\partial y^{2}}(x, y, u)\right|+\left|\frac{\partial^{2} L}{\partial y \partial u}(x, y, u)\right|+\left|\frac{\partial^{2} L}{\partial u^{2}}(x, y, u)\right| \leq C_{M}
$$

for a.e. $x \in \Omega$ and $|y|,|u| \leq M$. Finally, given $\rho>0$ arbitrary, for every $\varepsilon>0$ there exists $\delta>0$ such that for almost every point $x \in \Omega$ and $\left|y_{i}\right|,\left|u_{i}\right| \leq \rho, i=1,2$, we have

$$
\left|D_{(y, u)}^{2} L\left(x, y_{2}, u_{2}\right)-D_{(y, u)}^{2} L\left(x, y_{1}, u_{1}\right)\right|<\varepsilon \text { if }\left|y_{2}-y_{1}\right|<\delta,\left|u_{2}-u_{1}\right|<\delta,
$$

where $D_{(y, u)}^{2} L$ denotes the second derivative of $L$ with respect to $(y, u)$.

(H3) For every $1 \leq j \leq n_{e}+n_{i}, F_{j}$ is of class $C^{1}$ in $W^{1, s}(\Omega)$ and of class $C^{2}$ in $W^{1, q}(\Omega)$, where $s \in\left[1, \frac{N}{N-1}\right)$, $q \in\left[\max \left\{s, \frac{2 N}{N+2}\right\}, \frac{2 N}{N-2}\right)$, and $q \leq p$.

Let us show some examples of state constraints included in the previous formulation.

Example 4. Integral constraints on the state. Given $g_{j}: \Omega \times \mathbb{R} \longrightarrow \mathbb{R}$, we define $F_{j}(y)=\int_{\Omega} g_{j}(x, y(x)) d x$. Assumption (H3) is satisfied if we make the following hypotheses: $g_{j}$ is of class $C^{2}$ with respect to the second variable and measurable with respect to the first one, $g_{j}(\cdot, 0) \in L^{1}(\Omega)$, and for every $M>0$ there exist $\psi_{M} \in L^{N s /([N+1] s-N)}(\Omega)$, for some $s<N /(N-1)$, and $\psi_{M}^{*} \in L^{\alpha}(\Omega)$, with $\alpha=1$ if $N<4$ and $\alpha>N / 4$ otherwise, such that for every $y, y_{1}, y_{2} \in[-M,+M]$ and almost every $x \in \Omega$

$$
\left|\frac{\partial g_{j}}{\partial y}(x, y)\right| \leq \psi_{M}(x), \quad\left|\frac{\partial^{2} g_{j}}{\partial y^{2}}(x, y)\right| \leq \psi_{M}^{*}(x)
$$

$\forall \varepsilon>0 \exists \delta>0$ such that $\left|\frac{\partial^{2} g_{j}}{\partial y^{2}}\left(x, y_{2}\right)-\frac{\partial^{2} g_{j}}{\partial y^{2}}\left(x, y_{1}\right)\right| \leq \varepsilon \quad$ if $\left|y_{2}-y_{1}\right|<\delta$. 
(H3) holds for $q=\min \{p, 2 N /(N-2)-\beta\}>N$ for some $\beta>0$ small enough.

Example 5. Integral constraints on the derivatives of the state. Given $g_{j}: \Omega \times \mathbb{R}^{N} \longrightarrow \mathbb{R}$, we now define $F_{j}(y)=\int_{\Omega} g_{j}(x, \nabla y(x)) d x$. Then assumption (H3) is fulfilled if $g_{j}$ is of class $C^{2}$ with respect to the second variable and measurable with respect to the first one, $g_{j}(\cdot, 0) \in L^{1}(\Omega)$, there exist $C>0, r<2 p / N, \psi \in L^{s^{\prime}}(\Omega)$ for some $s<N /(N-1)$, and $\psi^{*} \in L^{\alpha}(\Omega)$ with $\alpha>N / 2$, such that

$$
\left|\frac{\partial g_{j}}{\partial \eta}(x, \eta)\right| \leq \psi(x)+C|\eta|^{p(s-1) / s}, \quad\left|\frac{\partial^{2} g_{j}}{\partial \eta^{2}}(x, \eta)\right| \leq \psi^{*}(x)+C|\eta|^{r}, \text { for a.e. } x \in \Omega,
$$

and finally, for every $M>0$ and $\varepsilon>0$ there exists $\delta=\delta(\varepsilon, M)>0$ such that

$$
\left|\frac{\partial^{2} g_{j}}{\partial \eta^{2}}\left(x, \eta_{2}\right)-\frac{\partial^{2} g_{j}}{\partial \eta^{2}}\left(x, \eta_{1}\right)\right| \leq \varepsilon \text { if }\left|\eta_{2}-\eta_{1}\right|<\delta \text { and }\left|\eta_{1}\right|,\left|\eta_{2}\right| \leq M, \text { for a.e. } x \in \Omega .
$$

Once again, (H3) is fulfilled for $q=\min \{p, 2 N /(N-2)-\beta\}$ for $\beta>0$ small enough. The reader is referred to $[8]$ for the study of this type of constraints.

The following result is proved in [7].

Theorem 6. Suppose that (H1) holds. Then for every $u \in L^{\infty}(\Omega)$ there exists a unique solution $y_{u} \in W^{1, p}(\Omega)$ of the state equation (1) and

$$
\forall M>0 \exists C_{M}>0 \text { such that }\left\|y_{u}\right\|_{W^{1, p}(\Omega)} \leq C_{M} \text { if }\|u\|_{L^{\infty}(\Omega)} \leq M .
$$

Now we check the differentiability of the functionals involved in the control problem; see also [7] for the proof.

Theorem 7. The functional $J: L^{\infty}(\Omega) \rightarrow \mathbb{R}$ is of class $C^{2}$. Moreover, for every $u, h \in L^{\infty}(\Omega)$

$$
J^{\prime}(u) h=\int_{\Omega}\left(\frac{\partial L}{\partial u}\left(x, y_{u}, u\right)+\varphi_{0 u} \frac{\partial f}{\partial u}\left(x, y_{u}, u\right)\right) h d x
$$

where $y_{u}=G(u), \varphi_{0 u} \in W^{1, p}(\Omega)$ is the unique solution of the problem

$$
\begin{gathered}
\left\{\begin{array}{cc}
-\Delta \varphi_{0 u}=\frac{\partial f}{\partial y}\left(x, y_{u}, u\right) \varphi_{0 u}+\frac{\partial L}{\partial y}\left(x, y_{u}, u\right) & \text { in } \Omega \\
\partial_{\nu} \varphi_{0 u}=0 & \text { on } \Gamma,
\end{array}\right. \\
J^{\prime \prime}(u) h^{2}=\int_{\Omega}\left[\frac{\partial^{2} L}{\partial y^{2}}\left(x, y_{u}, u\right) z_{h}^{2}+2 \frac{\partial^{2} L}{\partial y \partial u}\left(x, y_{u}, u\right) z_{h} h+\frac{\partial^{2} L}{\partial u^{2}}\left(x, y_{u}, u\right) h^{2}\right. \\
\left.+\varphi_{0 u}\left(\frac{\partial^{2} f}{\partial y^{2}}\left(x, y_{u}, u\right) z_{h}^{2}+2 \frac{\partial^{2} f}{\partial y \partial u}\left(x, y_{u}, u\right) z_{h} h+\frac{\partial^{2} f}{\partial u^{2}}\left(x, y_{u}, u\right) h^{2}\right)\right] d x
\end{gathered}
$$

where $z_{h}=G^{\prime}(u) h$ is the solution of Neumann problem

$$
\left\{\begin{aligned}
-\Delta z_{h} & =\frac{\partial f}{\partial y}\left(x, y_{u}, u\right) z_{h}+\frac{\partial f}{\partial u}\left(x, y_{u}, u\right) h & & \text { in } \Omega \\
\partial_{\nu} z_{h} & =0 & & \text { on } \Gamma .
\end{aligned}\right.
$$


Theorem 8. Functionals $G_{j}=F_{j} \circ G: L^{\infty}(\Omega) \rightarrow \mathbb{R}$ are of class $C^{2}$. Moreover, for every $u, h \in L^{\infty}(\Omega)$

$$
G_{j}^{\prime}(u) h=\int_{\Omega} \varphi_{j u} \frac{\partial f}{\partial u}\left(x, y_{u}, u\right) h d x
$$

and

$$
G_{j}^{\prime \prime}(u) h^{2}=F_{j}^{\prime \prime}\left(y_{u}\right) z_{h}^{2}+\int_{\Omega} \varphi_{j u}\left(\frac{\partial^{2} f}{\partial y^{2}}\left(x, y_{u}, u\right) z_{h}^{2}+\frac{\partial^{2} f}{\partial u^{2}}\left(x, y_{u}, u\right) h^{2}+2 \frac{\partial^{2} f}{\partial y \partial u}\left(x, y_{u}, u\right) z_{h} h\right) d x
$$

where $y_{u}=G(u), \varphi_{j u} \in W^{1, s^{\prime}}(\Omega)$ is the unique solution of the problem

$$
\left\{\begin{aligned}
-\Delta \varphi_{j u} & =\frac{\partial f}{\partial y}\left(x, y_{u}, u\right) \varphi_{j u}+F_{j}^{\prime}\left(y_{u}\right) & & \text { in } \Omega \\
\partial_{\nu} \varphi_{j u} & =0 & & \text { on } \Gamma,
\end{aligned}\right.
$$

and $z_{h}=G^{\prime}(u) h$

Using the previous theorems it is easy to check that Assumptions (A1)-(A3) are satisfied for the control problem. The Lagrangian function associated to the control problem is given by

$$
\mathcal{L}(u, \lambda)=J(u)+\sum_{j=1}^{m} \lambda_{j} G_{j}(u)=\int_{\Omega} L\left(x, y_{u}(x), u(x)\right) d x+\sum_{j=1}^{m} \lambda_{j} F_{j}\left(y_{u}\right)
$$

Let $\bar{u}$ be a local solution of the control problem satisfying (3) and let $\left\{\bar{\lambda}_{j}\right\}_{j=1}^{m}$ the Lagrange multipliers provided by Theorem 1 . Denoting by $\bar{\varphi}_{0}$ and $\bar{\varphi}_{j}$ the solutions of (23) and (24) corresponding to $\bar{u}$ and setting

$$
\bar{\varphi}=\bar{\varphi}_{0}+\sum_{j=1}^{n_{e}+n_{i}} \bar{\lambda}_{j} \bar{\varphi}_{j}
$$

we deduce from Theorems 7 and 8

$$
\begin{aligned}
\frac{\partial \mathcal{L}}{\partial u}(\bar{u}, \bar{\lambda}) h & =\int_{\Omega}\left(\frac{\partial L}{\partial u}(x, \bar{y}, \bar{u})+\bar{\varphi}_{0} \frac{\partial f}{\partial u}(x, \bar{y}, \bar{u})\right) h d x+\sum_{j=1}^{n_{e}+n_{i}} \bar{\lambda}_{j} \int_{\Omega} \bar{\varphi}_{j} \frac{\partial f}{\partial u}(x, \bar{y}, \bar{u}) h d x \\
& =\int_{\Omega}\left(\frac{\partial L}{\partial u}(x, \bar{y}, \bar{u})+\bar{\varphi} \frac{\partial f}{\partial u}(x, \bar{y}, \bar{u})\right) h d x=\int_{\Omega} d(x) h(x) d x \quad \forall h \in L^{\infty}(\Omega),
\end{aligned}
$$

where $\bar{y}=G(\bar{u})=y_{\bar{u}}$ and

$$
d(x)=\frac{\partial L}{\partial u}(x, \bar{y}(x), \bar{u}(x))+\bar{\varphi}(x) \frac{\partial f}{\partial u}(x, \bar{y}(x), \bar{u}(x))=\frac{\partial H}{\partial u}(x, \bar{y}(x), \bar{u}(x), \bar{\varphi}(x)),
$$

$H: \Omega \times \mathbb{R}^{3} \longrightarrow \mathbb{R}$ being the Hamiltonian associated to the control problem $(\mathrm{P})$,

$$
H(x, y, u, \varphi)=L(x, y, u)+\varphi f(x, y, u)
$$

¿From (5) we deduce that

$$
d(x)=\left\{\begin{array}{cl}
0 & \text { for a.e. } x \in \Omega, \text { where } u_{a}(x)<\bar{u}(x)<u_{b}(x) \\
\geq 0 & \text { for a.e. } x \in \Omega, \text { where } \bar{u}(x)=u_{a}(x) \\
\leq 0 & \text { for a.e. } x \in \Omega, \text { where } \bar{u}(x)=u_{b}(x)
\end{array}\right.
$$


Theorems 2, 3 and 4 as well as Corollary 1 are fulfilled under the assumption (3). Instead of using the abstracts results given in $\S 1$, we are going to use Pontryagin's principle for $(\mathrm{P})$ to deduce some second order optimality conditions. Pontryagin's principle for $(\mathrm{P})$ is formulated in terms of $H$ as follows.

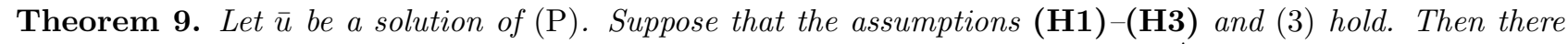
exist real numbers $\bar{\lambda}_{j}, j=1, \ldots, n_{i}+n_{e}$, and functions $\bar{y} \in W^{1, p}(\Omega), \bar{\varphi} \in W^{1, \min \left\{s^{\prime}, p\right\}}(\Omega)$ such that

$$
\begin{gathered}
\bar{\lambda}_{j} \geq 0, \quad n_{e}+1 \leq j \leq n_{e}+n_{i}, \quad \bar{\lambda}_{j} F_{j}(\bar{y})=0, \\
\left\{\begin{array}{cc}
-\Delta \bar{y}=f(x, \bar{y}(x), \bar{u}(x)) & \text { in } \Omega, \\
\partial_{\nu} \bar{y}=0 & \text { on } \Gamma
\end{array}\right. \\
\begin{cases}-\Delta \bar{\varphi}=\frac{\partial f}{\partial y}(x, \bar{y}, \bar{u}) \bar{\varphi}+\frac{\partial L}{\partial y}(x, \bar{y}, \bar{u})+\sum_{j=1}^{n_{e}+n_{i}} \bar{\lambda}_{j} F_{j}^{\prime}(\bar{y}) & \text { in } \Omega, \\
\partial_{\nu} \bar{\varphi}=0 & \text { on } \Gamma,\end{cases}
\end{gathered}
$$

and for a.e. $x \in \Omega$

$$
H(x, \bar{y}(x), \bar{u}(x), \bar{\varphi}(x))=\min _{k \in\left[u_{a}(x), u_{b}(x)\right]} H(x, \bar{y}(x), k, \bar{\varphi}(x))
$$

See [7] for the proof of this result. As an immediate consequence of Pontryagin's principle, Theorem 2, and Theorem 5, we obtain the first and second order necessary optimality conditions in the following way.

Corollary 2. Suppose that $\bar{u}$ is a local solution for problem (P). Suppose also that assumptions (H1)-(H3) and the regularity assumption (3) hold. Then there exist real numbers $\bar{\lambda}_{j}, j=1, \ldots, n_{i}+n_{e}$, and functions $\bar{y} \in W^{1, p}(\Omega), \bar{\varphi} \in W^{1, \min \left\{s^{\prime}, p\right\}}(\Omega)$ such that $(29)-(31)$ hold as well as the following relations:

$$
\begin{gathered}
\frac{\partial H}{\partial u}(x, \bar{y}(x), \bar{u}(x), \bar{\varphi}(x))(k-\bar{u}(x)) \geq 0 \text { for all } u_{a}(x) \leq k \leq u_{b}(x), \text { for a.e. } x \in \Omega, \\
\qquad \frac{\partial^{2} \mathcal{L}}{\partial u^{2}}(\bar{u}, \bar{\lambda}) h^{2} \geq 0 \text { for all } h \in C_{\bar{u}, L^{2}(\Omega)}^{0},
\end{gathered}
$$

and

$$
\frac{\partial^{2} H}{\partial u^{2}}(x, \bar{y}(x), \bar{u}(x), \bar{\varphi}(x)) \geq 0 \text { for a.e. } x \in \Omega \backslash \Omega^{0}
$$

In the above corollary $\Omega^{0}=X^{0}$ according to the notation $X=\Omega$; see (11).

In finite dimension, the first order optimality conditions and the strict positivity of the second derivative of the Lagrangian with respect to $u$ on $C_{\bar{u}}^{0}$ are sufficient conditions for a local minimum. The argument of the proof uses in an essential way the compactness of the balls in finite dimension. To extend this argumentation to infinite-dimensional optimization problems, Bonnans and Zidani [4] made the assumption that the second derivative of the Lagrangian with respect to $u$ was a Legendre form. Let us recall that a quadratic form $Q$ on a Hilbert space $\mathcal{H}$ is said to be a Legendre form if it is weakly lower semicontinuous, and for every sequence $\left\{x_{k}\right\} \subset \mathcal{H}$ that converges weakly $x_{k} \rightarrow x$ and such that $Q\left(x_{k}\right) \rightarrow Q(x)$, we have that $x_{k} \rightarrow x$ strongly. Unfortunately this assumption is not fulfilled, in general, in the context of control theory. We follow a different approach to achieve the same result. Along with the strict positivity of the second derivative of the Lagrangian, we assume that the second derivative of the Hamiltonian with respect to $u$ is strictly positive on $\Omega \backslash \Omega^{\tau}\left(\Omega^{\tau}=X^{\tau}\right.$ given by (17)), which is not far from the necessary condition provided in (35). More precisely, we have the following result proved in [7]. 
Theorem 10. Let $\bar{u}$ be an admissible control for problem $(\mathrm{P})$ satisfying $(\mathbf{H 1})-(\mathbf{H} 3)$, the regularity assumption (16), and (29)-(32) for some $\bar{\lambda}_{j}, j=1, \ldots, n_{i}+n_{e}$. Let us suppose also that there exist $\omega>0$ and $\tau>0$ such that

$$
\left\{\begin{array}{l}
\frac{\partial^{2} H}{\partial u^{2}}(x, \bar{y}(x), \bar{u}(x), \bar{\varphi}(x)) \geq \omega \text { for a.e. } x \in \Omega \backslash \Omega^{\tau}, \\
\frac{\partial^{2} \mathcal{L}}{\partial u^{2}}(\bar{u}, \bar{\lambda}) h^{2}>0 \text { for all } h \in C_{\bar{u}, L^{2}(\Omega)}^{0} \backslash\{0\} .
\end{array}\right.
$$

Then there exist $\varepsilon>0$ and $\alpha>0$ such that $J(\bar{u})+\alpha\|u-\bar{u}\|_{L^{2}(\Omega)}^{2} \leq J(u)$ for all admissible control $u$ with $\|u-\bar{u}\|_{L^{\infty}(\Omega)} \leq \varepsilon$.

Thus we have two different sufficient second order optimality conditions: those given by Corollary 1 and those established in Theorem 10. The next theorem shows the equivalence between both; see [7] for the proof.

Theorem 11. Let $\bar{u}$ be an admissible control for problem $(\mathrm{P})$ that satisfies $(\mathbf{H 1})-(\mathbf{H 3})$, the regularity assumption (3), and (29)-(32). Then the following two statements are equivalent:

(1) There exist $\delta>0$ and $\tau^{\prime}>0$ such that

$$
\frac{\partial^{2} \mathcal{L}}{\partial u^{2}}(\bar{u}, \bar{\lambda}) h^{2} \geq \delta\|h\|_{L^{2}(\Omega)}^{2} \text { for all } h \in C_{\bar{u}, L^{2}(\Omega)}^{\tau^{\prime}}
$$

(2) There exist $\omega>0$ and $\tau>0$ such that

$$
\left\{\begin{array}{l}
\frac{\partial^{2} H}{\partial u^{2}}(x, \bar{y}(x), \bar{u}(x), \bar{\varphi}(x)) \geq \omega \text { for a.e. } x \in \Omega \backslash \Omega^{\tau} \\
\frac{\partial^{2} \mathcal{L}}{\partial u^{2}}(\bar{u}, \bar{\lambda}) h^{2}>0 \text { for all } h \in C_{\bar{u}, L^{2}(\Omega)}^{0} \backslash\{0\} .
\end{array}\right.
$$

\section{REFERENCES}

[1] W. Alt and K. Malanowski. The lagrange-newton method for nonlinear optimal control problems. Comp. Optim. Appls., 2:77-100, 1993.

[2] N. Arada, E. Casas, and F. Tröltzsch. Error estimates for the numerical approximation of a semilinear elliptic control problem. Comp. Optim. Appls., 23(2):201-229, 2002.

[3] J.F. Bonnans and E. Casas. Contrôle de systèmes elliptiques semilinéaires comportant des contraintes sur l'état. In H. Brezis and J.L. Lions, editors, Nonlinear Partial Differential Equations and Their Applications. Collège de France Seminar, volume 8, pages 69-86. Longman Scientific \& Technical, New York, 1988.

[4] J.F. Bonnans and H. Zidani. Optimal control problems with partially polyhedric constraints. SIAM J. Control Optim., 37(6):1726-1741, 1999.

[5] H. Cartan. Calcul Différentiel. Hermann, Paris, 1967.

[6] E. Casas. Uniform convergence of the fem. applications to state constrained control problems. ESAIM:COCV, 8:345-374, 2002.

[7] E. Casas and M. Mateos. Second order optimality conditions for semilinear elliptic control problems with finitely many state constraints. SIAM J. Control Optim., 40(5):1431-1454, 2002.

[8] E. Casas, L.A. Fernández and M. Mateos. Second-order optimality conditions for semilinear elliptic control problems with constraints on the gradient of the state. Control Cybernet., 28(3):463-479, 1999.

[9] E. Casas and F. Tröltzsch. Second order necessary optimality conditions for some state-constrained control problems of semilinear elliptic equations. App. Math. Optim., 39:211-227, 1999.

[10] E. Casas and F. Tröltzsch. Second order necessary and sufficient optimality conditions for optimization problems and applications to control theory. SIAM J. Optim., 13(2):406-431, 2002.

[11] E. Casas, F. Tröltzsch, and A. Unger. Second order sufficient optimality conditions for a nonlinear elliptic control problem. $J$. for Analysis and its Appl., 15:687-707, 1996.

[12] E. Casas, F. Tröltzsch, and A. Unger. Second order sufficient optimality conditions for some state-constrained control problems of semilinear elliptic equations. SIAM J. Control Optim., 38(5):1369-1391, 2000.

[13] F.H. Clarke. A new approach to Lagrange multipliers. Math. Op. Res., 1(2):165-174, 1976. 
[14] A.L. Dontchev, W.W. Hager, A.B. Poore, and B. Yang. Optimality, stability, and convergence in nonlinear control. Appl. Math. Optim., 31:297-326, 1995.

[15] J.C. Dunn. Second-order optimality conditions in sets of $L^{\infty}$ functions with range in a polyhedron. SIAM J. Control Optim., 33(5):1603-1635, 1995.

[16] J.C. Dunn. On second order sufficient optimality conditions for structured nonlinear programs in infinite-dimensional function spaces. In A. Fiacco, editor, Mathematical Programming with Data Perturbations, pages 83-107. Marcel Dekker, 1998.

[17] H. Goldberg and F. Tröltzsch. Second order sufficient optimality conditions for a class of nonlinear parabolic boundary control problems. SIAM J. Control Optim., 31(4):1007-1025, 1993.

[18] W. Hager. Error bounds for euler approximation of a state and control constrained optimal control problem. Numer. Funct. Anal. and Optim., 21:653-682, 2000.

[19] K. Ito and K. Kunisch. Augmented lagrangian-sqp methods for nonlinear optimal control problems of tracking type. SIAM $J$. Control Optim., 34:874-891, 1996.

[20] H. Maurer. First and second order sufficient optimality conditions in mathematical programming and optimal control. Math. Programming Study, 14:163-177, 1981.

[21] H. Maurer and J. Zowe. First- and second-order conditions in infinite-dimensional programming problems. Math. Programming, 16:98-110, 1979.

[22] J.P. Raymond and F. Tröltzsch. Second order sufficient optimality conditions for nonlinear parabolic control problems with state-constraints. Discrete Contin. Dynam. Systems, 6:431-450, 2000. 